\title{
LA LEGISLACIÓN DE MEDIO AMBIENTE Y LA PROTECCIÓN DEL PATRIMONIO PALEONTOLÓGICO EN LA COMUNIDAD VALENCIANA (ESPAÑA)
}

\author{
Francisco Javier RUIZ SÁNCHEZ \\ Departament de Geologia. Facultat de Biologia. Universitat de València. Avda. \\ Dr. Moliner, 50.46100 Burjassot. e-mail: trancisco.ruiz@uv.es
}

Ruiz-Sánchez, F. J. 2005. La legislación de medio ambiente y la protección del patrimonio paleontológico en la Comunidad Valenciana (España). [The environmental legislation and paleontological heritage protection in the Valencian Community (Spain).] Revista Española de Paleontología, N.E. X, 119-124. ISSN 0213-6937.

\begin{abstract}
The paleontological heritage in the Valencian Community is rich and diverse, but it has been severely disturbed by human activities and it is in need of protection. The Spanish national law 4/1989 of conservation of natural sites, wild fauna and flora and the regional law 11/1994 of natural protected areas in the Valencian region (ESpain) consider the paleontological heritage as a natural resource. Paleontological sites of scientific and cultural interest may be proclaimed as natural protected areas after the approval of a management plan. The regional law 11/1994 states that paleontological resources fit into two protected area categories: Natural Monument and Site of Interest. The management plan for these two protection categories is named "protection norms". Law 11/1994 sets out the minimum contents of management plans including the restrictions to the use of natural resources in protected areas. Clearly, there is room for improvement in the way the regional authorities have protected and managed this heritage to date.
\end{abstract}

Keywords: Paleontological heritage, protection, environmental legislation, Valencian Community.

\section{RESUMEN}

Tanto en España, la Ley estatal 4/1989, de conservación de los espacios naturales y de la flora y fauna silvestres, como en la Comunidad Valenciana la Ley 11/1994, de espacios naturales protegidos, consideran el patrimonio paleontológico en la categoría de los recursos naturales. Los yacimientos paleontológicos con valores científicos o culturales de interés especial pueden ser declarados como espacios naturales protegidos a partir de un plan de ordenación de los recursos naturales. Según la Ley 11/1994, de la Generalitat Valenciana, de espacios naturales protegidos, los recursos paleontológicos pueden ser protegidos a través de la declaración de dos figuras de protección específicas, el Monumento Natural y el Sitio de Interés. El instrumento de ordenación para las figuras de Monumento Natural y Sitio de Interés se denomina normas de protección. La ley valenciana de espacios naturales establece el contenido mínimo de estos planes de ordenación, incluyendo las limitaciones de uso y disfrute de los recursos naturales en las zonas protegidas. El patrimonio paleontológico en la Comunidad Valenciana es rico y diverso, encontrándose negativamente afectado su estado de conservación por el intenso desarrollo de las actividades humanas. La gestión y protección de este recurso por parte de la administración valenciana puede claramente mejorarse.

Palabras clave: Patrimonio paleontológico, protección, legislación medioambiental, Comunidad Valenciana.

\section{INTRODUCCIÓN}

Las rocas que afl oran superfi cialmente en la Comunidad Valenciana son mayoritariamente sedimentarias. Las rocas ígneas y metamórficas están escasamente representadas. Por tanto, en el territorio de la Comunidad Valen- ciana existe una gran cantidad de afloramientos geológicos susceptibles de contener restos fósiles. En sus más de $23.000 \mathrm{~km}^{2}$ de superficie afloran materiales de todas las eras geológicas, a excepción de depósitos del Precámbrico. Son escasos los materiales aflorantes del Paleozoico, siendo mucho más abundantes y extensas las áreas ocupa- 
das por depósitos del Mesozoico y Cenozoico (Santanach et al., 1986; Sanchís et al., 1988; Guimerà et al., 1992). Los ambientes de depósito de estos materiales también son variados (Anadón et al., 2004). Así, se encuentran restos fósiles en depósitos formados en ambiente marino o continental. El registro sedimentario de algunas de las cuencas geológicas presenta alternancia entre depósitos marinos y continentales.

Si bien no todos estos afloramientos contienen restos fósiles, cada vez en mayor número de localidades se vienen realizando citas de taxones fósiles. El territorio de lo que hoy en día conforma la Comunidad Valenciana posee una flora y fauna fósil con una alta diversidad, fruto de unas peculiares características físicas del territorio valenciano a lo largo de su historia, además de por el hecho de haber estado situado en épocas anteriores en la encrucijada entre distintos sectores biogeográficos que posibilitaron el intercambio de especies entre uno y otro sector.

Sin pretender entrar en detalle, dado que no es el objetivo del presente trabajo, podemos decir que han podido ser recolectados restos pertenecientes a diferentes filums del registro fósil a lo largo y ancho de las diferentes comarcas de la geografía valenciana.

Todo ello conforma un variado y rico conjunto patrimonial paleontológico que progresivamente va aumentando en cantidad, encontrándose mucho de él expuesto a multitud de factores susceptibles de provocar su deterioro o desaparición.

El patrimonio paleontológico en la Comunidad Valenciana es incluido como valor patrimonial objeto de protección en la Ley 4/1998, de 11 de junio, de la Generalitat Valenciana, del Patrimonio Cultural Valenciano (Art. 1.2). Esta Ley establece la definición, forma de catalogación, régimen de protección y sancionador sobre los valores paleontológicos localizados en el territorio de la Comunidad Valenciana.

Esta vía de protección del patrimonio paleontológico no es la única con la que pueden contar las administraciones autonómicas en el Estado español. La segunda vía proteccionista se encuentra contemplada en la legislación medioambiental. Esta legislación, al igual que ocurre con la específica de Patrimonio, recoge como valor susceptible de ser protegido por su interés científico y natural el material paleontológico de notoria singularidad e interés. La legislación de protección del medio ambiente garantiza una protección de los recursos naturales contenidos en determinados espacios naturales. La figura del yacimiento paleontológico se establece como base sobre la que se apoya esta segunda vía proteccionista.

En el Estado español, la elaboración y ejecución de normas autonómicas para la protección del medio ambiente y de los valores que éste contiene parte de dos premisas básicas. Por un lado, la capacidad para elaborar, ejecutar y administrar normas propias proviene del grado compe- tencial del que se haya dotado la Comunidad Autónoma a través de su respectivo Estatuto de Autonomía (Aparicio, 1981), y por otro lado, depende de la existencia de una legislación estatal declarada como básica, y por tanto, de obligado cumplimiento en todo el estado. La Comunidad autónoma puede establecer su propia legislación al respecto, debiendo recoger ésta las cuestiones básicas así declaradas por la legislación estatal sectorial.

Así por tanto, el desarrollo legislativo y ejecución en materia de protección ambiental del patrimonio paleontológico en la Comunidad Valenciana deriva de lo establecido en su Estatuto de Autonomía (Ley Orgánica 5/1982, de 1 de julio, de Estatuto de Autonomía de la Comunidad Valenciana) y de la legislación estatal básica que, en materia de protección de espacios naturales, es la Ley 4/1989, de 27 de Marzo, de conservación de los espacios naturales y de la flora y fauna silvestre.

\section{CONSIDERACIONES}

La Ley 4/1989, de 27 de Marzo, de conservación de los espacios naturales y de la flora y fauna silvestre, surge de la necesidad y obligación de una utilización racional de todos los recursos naturales (artículo 45 de la Constitución). El mayor nivel de protección se aplica sobre las áreas que se definan como espacios naturales protegidos.

Como novedad en nuestro ordenamiento jurídico sobre protección del medio ambiente, la Ley estatal 4/1989 recoge la figura de los planes de ordenación de los recursos naturales (Art. 4.2), cuyos contenidos aseguran la planificación de los recursos naturales y constituyen un límite para cualquier otro instrumento de ordenación territorial o física, prevaleciendo sobre los existentes.

Así, podrá ser declarado como espacio protegido, cualquier área del territorio nacional que contenga elementos o valores naturales sobresalientes o de especial interés, con el fin de proteger elementos naturales de interés singular desde el punto de vista científico, cultural, educativo, etc (Art. 10.1 y 10.2). En función de los valores y bienes a proteger, la Ley establece cuatro categorías de espacios naturales protegidos: Parque, Reserva Natural, Monumento natural y Paisaje protegido. Los yacimientos paleontológicos de interés especial se incluyen dentro de la figura de monumentos naturales (Art. 16.2).

\section{LEGISLACIÓN AUTONÓMICA VALENCIANA}

El Estatuto de autonomía de la Comunidad Valenciana dota a la Generalitat Valenciana de competencia exclusiva sobre la protección de espacios naturales, según lo dispuesto en la Carta Magna (Art. 149.1.23). 
El artículo 149.1.23 de la Constitución española reserva al estado la competencia exclusiva para dictar la legislación básica sobre protección del medio ambiente, a partir de la cual las comunidades autónomas podrán elaborar, ejecutar y administrar las medidas de conservación de la naturaleza que estatutariamente les competan.

Dentro de este ámbito competencial, y siguiendo los criterios básicos contenidos en la citada Ley 4/1989, y teniendo en cuenta, además, los contenidos de la Directiva europea 92/43/CE, de Hábitats, las Cortes Valencianas aprobaron una Resolución recabando del gobierno valenciano la presentación de un proyecto de ley de espacios naturales protegidos de la Comunidad Valenciana durante el año 1994. Esta Ley autonómica sustituiría a la precedente, de 24 de junio, reguladora de los parajes naturales de la Comunidad Valenciana. Fruto de esta acción es promulgada la Ley 11/1994, de 27 de diciembre, de la Generalitat Valenciana, de espacios naturales protegidos de la Comunidad Valenciana.

La citada Ley recoge, al igual que su homóloga estatal, como elementos o valores merecedores de protección al que podríamos denominar desde una óptica amplia como "recurso paleontológico". Este recurso debe ser entendido como todos aquellos valores o elementos de la naturaleza que, de forma directa o indirecta, nos aportan información acerca de las biotas que, desde el pasado hasta la actualidad, han poblado la biosfera de la Tierra.

En la legislación valenciana de espacios naturales protegidos, el recurso paleontológico también puede ser incluido como factor clave para la declaración de un área como protegida. Además, mientras la ley estatal 4/1989 contempla a la figura de Monumento Natural como válida para la protección de valores paleontológicos sobresalientes y de interés, la Ley 11/1994 valenciana incluye una nueva figura de protección, el Sitio de Interés.

Según la Ley autonómica 11/1994, son monumentos naturales los espacios o elementos de la naturaleza, incluidas las formaciones geomorfológicas y yacimientos paleontológicos, de notoria singularidad, rareza o belleza, que merecen ser objeto de una protección especial por sus valores científicos, culturales o paisajísticos (Art. 11.1). Por su parte, son sitios de interés los enclaves territoriales en que concurran valores merecedores de protección por su interés para las ciencias naturales (Art. 12.1).

De igual modo que la legislación estatal, la legislación valenciana de espacios protegidos adopta el mecanismo de planificación de los recursos como el más adecuado para cumplir los objetivos fijados. Cabe destacar, no obstante, una diferencia significativa, fruto de la competencia en el desarrollo legislativo, como es la adecuación en denominación y contenidos de los instrumentos de ordenación a la figura de protección que vaya a ser declarada.

Para conseguir los objetivos de ordenación previstos, la legislación autonómica establece un mecanismo abre- viado y otro "in extenso", en función del tipo de figura de protección con que vaya a ser declarado el espacio. Esta forma de planificar es muy similar a la forma de evaluar contenida en la legislación autonómica valenciana de evaluación de impacto ambiental, donde se adopta un tipo abreviado o extenso según la magnitud del proyecto objeto de evaluación.

Así, la Ley 11/1994 establece cuatro instrumentos de ordenación y planificación ambiental. Estos son los planes de ordenación de los recursos naturales (PORN), los planes rectores de uso y gestión (PRUG), los planes especiales y las normas de protección.

\section{LAS NORMAS DE PROTECCIÓN}

El instrumento de ordenación elegido para las figuras de Monumento Natural y Sitio de Interés es el de las normas de protección. Este instrumento es el que establece el régimen de usos y actividades permisibles y las limitaciones aplicables al entorno de estos espacios. Se trata de uno de los mecanismos abreviados de ordenación recogidos en la legislación autonómica.

Para las normas de protección, la Ley 11/1994 establece un contenido mínimo que puede ser ampliado en función de los valores a proteger y de las circunstancias peculiares del espacio. El contenido mínimo incluye cuatro aspectos básicos: 1) la delimitación del ámbito geográfico de protección, que podrá ser discontinuo cuando resulte necesario, 2) la identificación de los valores a proteger y los posibles riesgos que pueda ponerlos en peligro, 3) las normas de uso y aprovechamiento del suelo y los recursos naturales, destinadas a proteger y conservar o mejorar los valores ambientales, y 4) las normas relativas a la visita o uso público, así como a las actividades científicas y educativas.

Con carácter general, la declaración de un espacio natural protegido comporta la declaración de utilidad pública e interés social de todos los bienes y derechos dentro del área protegida, incluidos los expropiatorios, el sometimiento de las transmisiones de terrenos a los derechos de tanteo y retracto, la sujeción a la servidumbre de instalación de señales (informativas, de situación, etc.) y la utilización de estos bienes con arreglo a lo previsto por la Ley valenciana de espacios naturales protegidos y sus instrumentos de ordenación.

\section{ESTADO ACTUAL DE LA PROTECCIÓN DEL RECURSO PALEONTOLÓGICO EN LA COMUNIDAD VALENCIANA. SITUACIÓN ADMINISTRATIVA}

La declaración de un espacio natural protegido puede realizarse tras un proceso de ordenación exhaustivo don- 
de no existan perturbaciones que hagan peligrar el recurso natural objeto de protección, o mediante la vía de urgencia en caso de existir algún factor que amenaza o altera al recurso.

En este sentido, la acción antrópica suele constituirse como principal factor de amenaza. Esta acción puede resultar de una falta de planificación o de una planificación deficiente donde no se tiene en cuenta el recurso paleontológico. Un ejemplo de "planificación deficiente", que puede afectar negativamente a recursos paleontológicos de notoria singularidad e interés, lo encontramos en la pretensión de instalación de una planta de tratamiento de residuos inertes en el área de yacimientos paleontológicos del Plioceno - Pleistoceno de Casablanca (Almenara). Como alguno de nuestros colegas ha participado a la comunidad paleontológica española en las últimas jornadas de paleontología de Morella, este proyecto, de llevarse a término, haría peligrar la conservación de este singular enclave (Furió et al., 2005).

Como instrumento eminentemente preventivo, la simple aplicación de la legislación de evaluación de impacto ambiental debería ser suficiente para atajar situaciones como las arriba comentadas para los yacimientos de Casablanca. Esto es, un proyecto de instalación de un vertedero, aparte de estar incluido en un plan provincial de manejo de residuos, debe ser y es objeto de una declaración de impacto ambiental por parte de la administración medioambiental autonómica. En este proceso de evaluación, y con carácter previo a la declaración de impacto, que establecerá la idoneidad y límites de acción del proyecto, o su rechazo, los diferentes departamentos de la administración deben informar preceptivamente acerca de las posibles repercusiones del proyecto sobre los temas de su competencia.

A veces los procedimientos no son tan ordenados y eficaces como cabría esperar. Así, la corriente falta de información y capacidad de gestión de la propia administración, provoca situaciones donde el recurso natural queda desprotegido o expuesto a su deterioro.

Para estas y otras situaciones, la legislación sectorial se suele reservar la posibilidad de retrotraer o hacer prevalecer unas normas sobre otras previamente dictadas. Esto es lo que puede ocurrir de aplicarse la legislación de espacios naturales protegidos. Así, la Ley valenciana 11/1994 recoge, por un lado, que las normas de protección de monumentos naturales y sitios de interés tendrán carácter vinculante, tanto para las administraciones públicas como para los particulares; por otro, que las normas de protección prevalecerán sobre el planeamiento urbanístico y, por último, que la aprobación de las normas de protección conllevará necesariamente la revisión de oficio de los planes territoriales sectoriales incompatibles con las mismas.

Los contenidos de las leyes estatales y autonómica de espacios naturales protegidos, con sus correspondientes reservas cautelares, deberían ser, junto a los preceptos re- cogidos en la Ley 4/1998, de 11 de junio, de la Generalitat Valenciana, del Patrimonio Cultural Valenciano, suficientes para garantizar una adecuada protección de los valores paleontológicos en el ámbito de la Comunidad Valenciana. No obstante, la realidad no es tan halagüeña como cabría esperar del abundante acervo legislativo de protección con que se cuenta.

\section{SITUACIÓN ADMINISTRATIVA}

La protección del recurso paleontológico en el ámbito de la Comunidad Valenciana se puede realizar a través de dos organismos de la Generalitat Valenciana con competencias en la materia. Por un lado, la Consejería de Cultura, Educación y Deporte, responsable de las competencias de patrimonio histórico y, por otro, de la Consejería de Territorio y Vivienda, competente en materia de espacios naturales protegidos.

A nuestro juicio, la protección del patrimonio paleontológico se debe sustentar en dos pilares básicos. Por un lado, a partir del conocimiento del valor objeto de protección, y por otro a través de una correcta gestión de las administraciones competentes. Actualmente, la Consejería de Cultura, Educación y Deporte cuenta con una línea de subvenciones que persiguen como objetivo acrecentar y poner en valor los recursos paleontológicos a partir de excavaciones paleontológicas, restauración y catalogación de materiales paleontológicos, lo que ayuda a cumplir, en la medida de las posibilidades, con la primera premisa proteccionista, el conocimiento del patrimonio paleontológico. Por su parte, el sector medioambiental de la administración valenciana no promueve ningún tipo de estudio propio o línea de subvención en este sentido.

Las competencias en protección del recurso paleontológico de la administración ambiental valenciana quedan adscritas a la Consejería de Territorio y Vivienda. Después de ocho años de vigencia de la Ley 11/1994, de espacios naturales protegidos de la Comunidad Valenciana, no se ha declarado ni un sólo espacio bajo las figuras de Monumento Natural o Sitio de Interés. Es curioso destacar que entre los técnicos de la administración de medio ambiente de la Comunidad Valenciana existe personal cuya preparación biológica y geológica es más que adecuada para las labores de protección del recurso paleontológico. Sin embargo, en los más de quince años de existencia de la administración medioambiental valenciana no se ha realizado trabajo alguno relacionado con la temática de protección paleontológica. La actuación política y administrativa de la administración ambiental queda circunscrita únicamente a la protección de los recursos biológicos vivos, relegando a la protección de los recursos paleontológicos al papel impreso de la Ley, dejando carente de contenidos las buenas intenciones recogidas en la propia Ley. 
Lo cierto es que ninguno de los quince Espacios Naturales Protegidos de la Comunidad Valenciana actualmente existentes justifica su declaración en la conservación de su contenido paleontológico. Son numerosas las situaciones que demandan una urgente acción preventiva y proteccionista del recurso paleontológico por parte de la administración medioambiental valenciana. Ejemplo de estas situaciones son las zonas declaradas o a punto de ser declaradas como vertederos de residuos. Anteriormente se ha comentado el lacerante caso de los yacimientos de vertebrados de las canteras de Casablanca. El caso del yacimiento de plantas fósiles de Olocau, salvado "in extremis", por el momento, por la denuncia de personal del Departamento de Geología de la Universitat de València, o el más que preocupante plan de arcillas de la Comunidad Valenciana, fruto del acuerdo del gobierno valenciano con la potente industria cerámica de la Comunidad para abastecer de materias primas a esta última, y otros más, se suman para vislumbrar un presente y un futuro nada halagüeños.

\section{CONCLUSIONES}

El territorio de la Comunidad Valenciana cuenta con un patrimonio paleontológico de primer orden en el que se registran biotas fósiles de una parte considerable de los filums conocidos en el periodo comprendido entre el inicio del Mesozoico y la actualidad.

El recurso paleontológico en el Estado español puede ser objeto de protección a través de la legislación de patrimonio histórico y de espacios naturales protegidos, que en la Comunidad Valenciana se concreta en las leyes 4/1998, de 11 de junio, de la Generalitat Valenciana, del Patrimonio Cultural Valenciano, y en la Ley 11/1994, de 27 de diciembre, de la Generalitat Valenciana, de Espacios Naturales Protegidos de la Comunidad Valenciana, respectivamente.

La Ley valenciana 11/1994 basa la protección del recurso paleontológico en un proceso mixto de ordenación y declaración como espacio natural protegido. Para el recurso paleontológico, la citada Ley contempla, básicamente, las figuras de Monumento Natural y Sitios de Interés. Sin embargo, esta Ley no ha sido aplicada por el momento para la protección de ningún área que contenga recursos paleontológicos.

En la Comunidad Valenciana, algunas zonas con importantes recursos paleontológicos se encuentran incluidas en el área de actuación de planes territoriales sectoriales que, en un futuro cercano, pueden provocar un grave deterioro o destrucción de recursos paleontológicos de gran interés científico y cultural.

$\mathrm{Al}$ igual que ocurre en los sitios de interés arqueológico, los estudios de impacto ambiental de los proyectos en cuyas zonas de actuación existan o puedan existir recursos paleontológicos, deben contar con un apartado en el que se caractericen adecuadamente estos. La realización y evaluación administrativa de estos estudios debe ser realizada por personal con capacidad técnica reconocida.

El hecho de tener carácter vinculante, y de prevalecer los contenidos de las normas de protección sobre cualquier otro planeamiento territorial existente, hacen de la declaración como Monumento Natural o Sitio de Interés un mecanismo adecuado de protección del recurso paleontológico en aquellas áreas donde estén, o puedan encontrarse, afectados por perturbaciones que amenacen su preservación y buen estado de conservación.

La inclusión del recurso paleontológico como otra modalidad más de recurso natural, el actual estado de conservación y las amenazas que se ciernen sobre éste hacen necesaria la implicación de la administración medioambiental en su protección. Urge, por tanto, la concienciación política sobre el tema y la asignación de medios humanos y económicos que palien el hecho que, la no aplicación de la legislación de espacios naturales protegidos para las áreas con recursos paleontológicos de interés, merma sensiblemente la capacidad de protección de este recurso en la Comunidad Valenciana.

\section{BIBLIOGRAFÍA}

Anadón Monzón, P., Alcalá, L., Alonso-Zarza, A.M., Calvo, J.P., Ortí, F., Rosell, L. \& Sanz-Rubio, E. 2004. Cuencas de la Cordillera Ibérica. In: Geología de España (Ed. J.A. Vera). Sociedad Geológica de España - Instituto Geológico y Minero de España, Madrid, 562-569

Aparicio, M. A. 1981. Introducción al sistema político y constitucional español. Editorial Ariel, Barcelona, 180 pp.

Furió, M., Santos-Cubedo, A., Hugues-Alexandre, B., Blaya, E., Casanovas-Vilar, I., Madurell, J., Gusi, F. y Martínez-Alba, D. 2005. Síntesis sobre las faunas fósiles del complejo cárstico Almenara - Casablanca (Castellón). Publicaciones del Seminario de Paleontología de Zaragoza, 6, 273-286.

Guimerá, J., Anadón, P., Cabrera, L., Estévez, A., Martí, J. \& Taberner, C. 1992. Geología II. In: Enciclopèdia d'Història Natural dels Països Catalans. Enciclopèdia Catalana S.A., Barcelona, 548 pp.

Ruiz-Sánchez, F. J. 2003. La protección del patrimonio paleontológico en la legislación de medio ambiente de la Comunidad Valenciana. Libro de resúmenes de las XIX Jornadas de Paleontología. Morella 2003, 152-153.

Sanchís Moll, E. J., Rodríguez Estrella, T. \& Morell Evangelista, I. 1988. Geología. In: Guía de la naturaleza de la Comunidad Valenciana (Dir. E. J. Sanchos Moll). Ediciones Alfonso el Magnánimo - Diputació Provincial de València - Generalitat Valenciana, Valencia, 19-72.

Santanach, P., Banda, E., Calvet, F., Carreras, J., Casas, J. M., Fontboté, J. M., Fornós, J., Gisbert, J., Julivert, M., 
Marzo, M., Rodríguez-Perea, A., Salas, R. \& Bouquet, P. 1986. Geologia I. In: Enciclopèdia d'Història Natural dels Països Catalans. Enciclopèdia Catalana S.A., Barcelona, $439 \mathrm{pp}$.

\section{REFERENCIAS LEGISLATIVAS}

Ley Orgánica 5/1982, de 1 de julio, de estatuto de autonomía de la Comunidad Valenciana. (B.O.E. $\mathrm{n}^{\circ}$ 164, 10/07/1982).
Ley 4/1989, de 27 de marzo, de conservación de los espacios naturales y de la flora y fauna silvestres. (B.O.E. $\mathrm{n}^{\circ} 74$, 28/03/1989).

Ley 11/1994, de 27 de diciembre, de la Generalitat Valenciana, de espacios naturales protegidos de la Comunidad Valenciana. (D.O.G.V. no 2423, 09/01/1995).

Ley 4/1998, de 11 de junio, de la Generalitat Valenciana, del patrimonio cultural valenciano. (D.O.G.V. $\mathrm{n}^{\circ} 3267$, 18/06/1998).

Manuscrito recibido: 14 de Enero, 2004 Manuscrito aceptado: 27 de Mayo, 2005 